\title{
Effect of oligofructose supplementation on body weight in overweight and obese children: a randomised, double-blind, placebo-controlled trial
}

\author{
Anna Liber* and Hania Szajewska \\ Department of Paediatrics, The Medical University of Warsaw, Dzialdowska 1, 01-184 Warsaw, Poland \\ (Submitted 27 June 2014 - Final revision received 15 August 2014 - Accepted 26 August 2014 - First published online 20 October 2014)
}

\begin{abstract}
Limited evidence suggests that the dietary inclusion of oligofructose, an inulin-type fructan with prebiotic properties, may increase satiety and, thus, reduce energy intake and body weight in overweight and obese adults. The aim of the present study was to assess the effect of oligofructose supplementation for 12 weeks on the BMI of overweight and obese children. A total of ninety-seven children aged 7-18 years who were overweight and obese (BMI $>85$ th percentile) were randomly assigned to receive placebo (maltodextrin) or oligofructose (both at an age-dependent dose: $8 \mathrm{~g} / \mathrm{d}$ for children aged 7-11 years and 15 g/d for children aged 12-18 years) for $12 \mathrm{weeks}$. Before the intervention, all children received dietetic advice and they were encouraged to engage in physical activity. The primary outcome measure was the BMI-for-age $z$-score difference between the groups at the end of the intervention. Data from seventy-nine ( $81 \%)$ children were available for analysis. At 12 weeks, the BMI-for-age $z$-score difference did not differ between the experimental ( $n$ 40) and control ( $n$ 39) groups (mean difference $0 \cdot 002,95 \%$ CI $-0 \cdot 11,0 \cdot 1$ ). There were also no significant differences between the groups with regard to any of the secondary outcomes, such as the mean BMI-for-age $z$-score, percentage of body weight reduction and the difference in total body fat. Adverse effects were similar in both groups. In conclusion, oligofructose supplementation for 12 weeks has no effect on body weight in overweight and obese children.
\end{abstract}

\section{Key words: Inulin-type fructans: Prebiotics: Obesity}

The prevalence of obesity is now reaching epidemic proportions worldwide, stimulating research to identify factors that affect energy balance ${ }^{(1)}$. Current evidence suggests a link between gut microbiota and obesity ${ }^{(2)}$. It has been shown that there are differences between the gut microbiota of lean and obese individuals, suggesting the importance of the Firmicutes:Bacteroidetes ratio $^{(3)}$, although the data are conflicting. Moreover, recent studies suggest the diet to have an impact on the composition of the gut microbiota (showing that dietary manipulation modifies bacterial profiles and metabolism $)^{(4)}$. Furthermore, the development of obesity may be influenced by the exposure to a bacterial endotoxin (lipopolysaccharide) derived from the gut microbiota ${ }^{(5)}$. The resulting metabolic endotoxaemia is thought to contribute to low-grade inflammation, a characteristic of obesity and the metabolic syndrome. Although causality remains to be confirmed, taken together, these findings support the view that the gut microbiota plays some role in the development of obesity. If so, it is logical to assume that manipulation of the gut microbiota, e.g. through the administration of prebiotics and/or probiotics, could potentially be a preventive and/or therapeutic measure in the evolution of obesity.
Oligofructose, an inulin-type fructan, is a non-digestible, fully soluble and fermentable carbohydrate, with known prebiotic properties, which is found naturally in chicory, onion and garlic ${ }^{(6)}$. Data from previous animal studies have shown that a high-fat diet enriched with oligofructose leads to a decrease in energy intake, less weight gain, and a lower concentration of $\mathrm{TAG}^{(7)}$. The mechanism by which oligofructose may influence body weight remains unclear. However, it has been shown that oligofructose fermentation modulates the secretion of gastrointestinal hormones (i.e. glucagon-like peptide-1 and glucagon-like peptide-2), thus promoting satiety, and decreases the concentration of ghrelin, which stimulates food intake ${ }^{(7,8)}$. Moreover, two human trials have reported that oligofructose consumption increases the concentrations of satiety hormones ${ }^{(9,10)}$.

We have recently carried out a systematic review of randomised controlled trials (RCT) to evaluate the effects of inulin-type fructan supplementation, including oligofructose, on appetite, energy intake and body weight in children and adults. Limited data suggest that the long-term supplementation of inulin-type fructans may contribute to weight reduction in adolescents and in overweight and obese adults ${ }^{(11)}$. Beneficial

Abbreviations: DXA, dual-energy X-ray absorptiometry; MD, mean difference; RCT, randomised controlled trials; RR, relative risk.

*Corresponding author: A. Liber, fax +48 2245233 09, email anna.liber@wp.pl 
effects of inulin-type fructans on metabolic disorders associated with obesity, i.e. glucose intolerance and dyslipidaemia, have been investigated in many studies, but the results of these studies are inconsistent ${ }^{(12,13)}$. As inulin-type fructans are not homogeneous, further RCT are recommended to determine the type of substance that is the most effective. Furthermore, studies in children are scarce and more studies are needed. The present study aimed to assess the effect of oligofructose supplementation for 12 weeks on the BMI of overweight and obese children. The hypothesis was that oligofructose supplementation would reduce BMI. The choice of oligofructose was determined based on the findings from an earlier study carried out in adults ${ }^{(14)}$.

\section{Methods}

This is a randomised, double-blind, placebo-controlled trial that was initiated by the investigators and was conducted independently of any commercial entities. The trial protocol was registered at ClinicalTrials.gov (NCT01673152). The trial was conducted between January 2012 and December 2013 at the Department of Paediatrics, the Medical University of Warsaw, Poland. Children aged $7-18$ years with a BMIfor-age $>85$ th percentile were eligible to participate in the study. Those with a BMI-for-age between 85th and 97th percentiles (equivalent to $>1 \mathrm{sD}$ according to WHO criteria $^{(15)}$ ) were classified as overweight and those with a BMI-for-age $>97$ th percentile (equivalent to $>2$ sD according to WHO criteria) were classified as obese. Exclusion criteria included overweight/obesity secondary to genetic syndromes, endocrine diseases, and the use of drugs that influence appetite or body weight. Eligible children were randomly assigned to receive oligofructose (Orafti P95, Beneo-Orafti) or a comparable placebo (maltodextrin). Both oligofructose and placebo were prepared in identical sachets by the hospital pharmacy. Dosing of oligofructose was age dependent. Children aged 7-11 years received $8 \mathrm{~g}$ daily and those aged 12-18 years received $15 \mathrm{~g}$ daily. Maltodextrin was dosed in equienergetic amounts. The study products were administered twice daily orally for 12 weeks. The participants were advised to dissolve the study products in water and to take them preferably before breakfast and dinner. Data from previous studies were used when making decisions regarding dosing $^{(16,17)}$, the duration of the intervention ${ }^{(14)}$ and the choice of placebo ${ }^{(14)}$. All parents and children received the same dietary advice from the dietitian. The participants were advised to follow a normoenergetic diet throughout the study. The Nutrition Standards for Polish population developed by the National Food and Nutrition Institute ${ }^{(18)}$ were used to calculate energy considering the participant's age and low level of physical activity. All children were encouraged to engage in physical activity for at least $1 \mathrm{~h}$ daily.

At study entry, all eligible children underwent a physical examination, including evaluation of blood pressure and anthropometric measurements (weight, height and BMI, which were plotted on WHO 2007 growth curves; http:// www.who.int/growthref/en/). The laboratory examinations were performed at baseline using standard methods at the hospital laboratory of the Medical University of Warsaw and included measurements of fasting glucose, total cholesterol, LDL-cholesterol, HDL-cholesterol and TAG. Total body fat was measured by dual-energy X-ray absorptiometry (DXA) using Lunar Prodigy (GE Healthcare). Each participant maintained a $3 \mathrm{~d}$ food record over 2 weekdays and 1 weekend day, which was then reviewed by the dietitian using the computer software DIETA 5.0; http://www.izz.waw.pl (National Food and Nutrition Institute, Warsaw, 2011). Physical activity was assessed using the International Physical Activity Questionnaire (described in detail elsewhere ${ }^{(19)}$ ), which earlier was modified and adopted for children ${ }^{(20)}$. To assess tolerance to the study products, children were asked to fill in a diary in which listed symptoms, primarily gastrointestinal symptoms such as abdominal pain and flatulence, were reported. In addition, children were encouraged to report any other symptoms on their own initiative.

At the end of the intervention period (i.e. at 12 weeks), anthropometric measurements were performed again. Total body fat was measured using DXA. A $3 \mathrm{~d}$ food record, an activity questionnaire and a diary reporting adverse effects were obtained from the participants. The laboratory examinations were repeated only in case of abnormalities at baseline. To assess compliance, the participants were asked to return empty sachets. At the end of the follow-up period (i.e. at 24 weeks), only anthropometric measurements were performed.

The primary outcome measure was the BMI-for-age $z$-score difference between the groups at 12 weeks (baseline $v$. end of the intervention). The secondary outcome measures at 12 weeks included the mean BMI-for-age $z$-score; percentage of body weight reduction; difference in total body fat measured by DXA; number of children with a BMI-for-age $<85$ th percentile; self-reported energy intake; number of children with abnormal fasting glucose concentrations $(>6.11 \mathrm{mmol} / \mathrm{l}$; $>110 \mathrm{mg} / \mathrm{dl})^{(21)}$; number of children with dyslipidaemias (i.e. total cholesterol concentrations $>5.18 \mathrm{mmol} / \mathrm{l}(>200 \mathrm{mg} / \mathrm{dl})$, LDL-cholesterol concentrations $>3.37 \mathrm{mmol} / \mathrm{l}(>130 \mathrm{mg} / \mathrm{dl})$, HDL-cholesterol concentrations $<0.91 \mathrm{mmol} / \mathrm{l} \quad(<35 \mathrm{mg} / \mathrm{dl})$ and TAG concentrations $>1.70 \mathrm{mmol} / 1(>150 \mathrm{mg} / \mathrm{dl}))^{(22)}$; percentage of children with hypertension (according to the criteria of the European Society of Hypertension $)^{(23)}$; physical activity; and adverse effects. The secondary outcomes measured at 24 weeks (end of the follow-up period) included the BMI-for-age $z$-score difference; the mean BMI-for-age $z$-score; and the number of children with a BMI-for-age $<85$ th percentile.

Randomisation was based on computer-generated numbers and was performed in blocks of six. The randomisation list was prepared by an investigator with no clinical involvement in the study. The randomisation sequence and codes were secured until all data were analysed. All participants and investigators were blinded to the assigned treatment throughout the study. The study products were white powders similar in terms of texture, smell and colour and were packaged in identical sachets. To ensure concealment, the study products were weighed, packaged and signed by consecutive number according to the randomisation list generated by the hospital pharmacy at the Medical University of Warsaw by independent personnel not involved in the conduct of the study. 


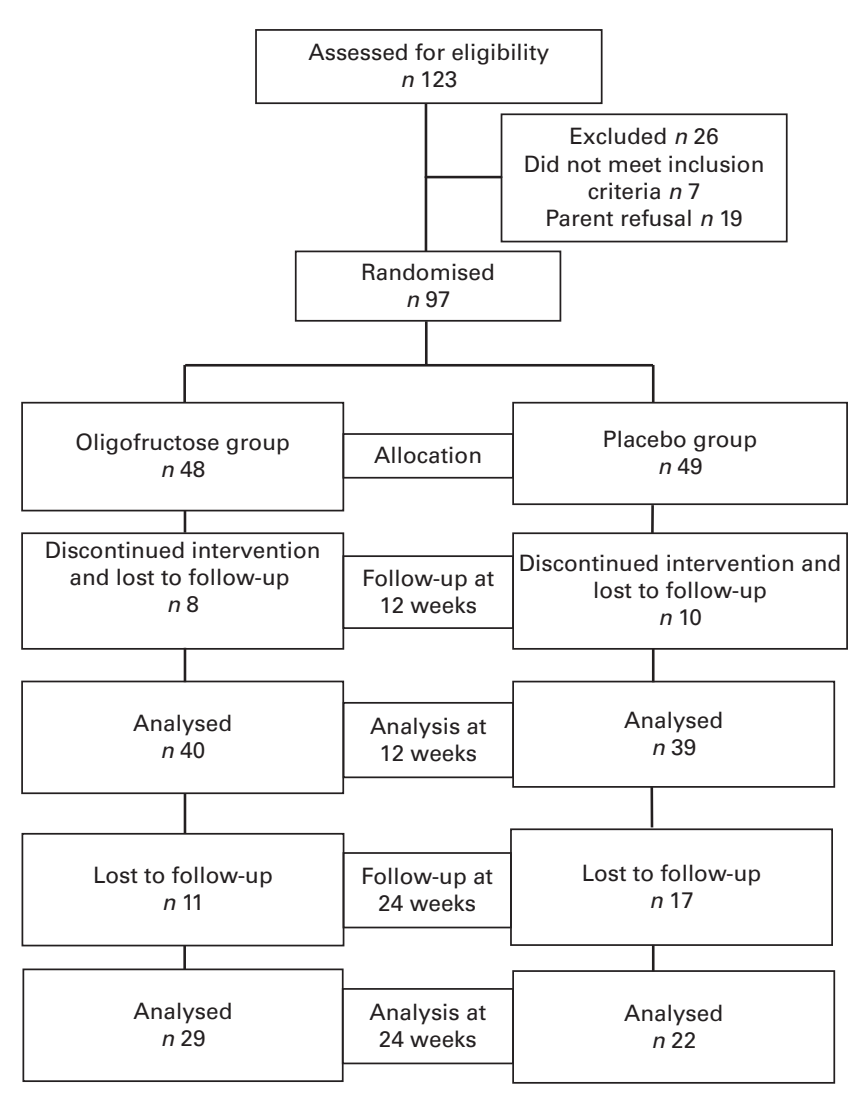

Fig. 1. Flowchart depicting the progression of participants through the study.

\section{Sample size}

The primary endpoint was the difference in BMI-for-age $z$-scores between the groups. Considering data from the literature ${ }^{(24)}$, it was assumed that the mean difference (MD) would be $0 \cdot 17$, with a SD of $0 \cdot 267$. To detect this difference, with a power of $80 \%$ and a significance level of $5 \%$ and taking into account that $20 \%$ of the patients will be lost to followup, it was calculated that forty-eight patients would be needed to be studied in each group.

\section{Statistical analyses}

Student's $t$ test was used to compare the mean values of continuous variables approximating a normal distribution. The Mann-Whitney $U$ test was used to compare the mean values of non-normally distributed variables. Data that were not normally distributed are reported as medians and ranges. The $\chi^{2}$ test was used to compare percentages. Differences between the study groups were considered significant when the $P$ value was $<0.05$, when the $95 \%$ CI for the MD did not include 0 , or when the $95 \% \mathrm{CI}$ for the relative risk (RR) did not include 1 (equivalent to $P<0.05$ ). The analyses were carried out based on allocated treatment, i.e. all the participants in a trial for which outcome data were available were analysed according to the intervention that they were assigned, whether or not they received it. Statistical analyses were performed using the data analysis software system STATISTICA (version 10, 2011; StatSoft, Inc.; http://www.statsoft.com).

\section{Ethical approval}

The present study was conducted according to the guidelines laid down in the Declaration of Helsinki, and all procedures involving human subjects were approved by the ethics committee of the Medical University of Warsaw. Written informed consent was obtained from at least one parent and from children aged $\geq 16$ years.

\section{Results}

A flowchart depicting the progression of participants through the study is shown in Fig. 1. Of the 123 eligible children, ninety-seven underwent randomisation. Among them, fortyeight were assigned to the experimental oligofructose group and forty-nine were assigned to the placebo group. At 12 weeks (end of the intervention), data from seventy-nine (81\%) children were available for analysis. Dropouts comprised children who did not arrive for the study visit and were lost to follow-up. The dropout rates were similar in both groups. None of the dropouts had reported adverse effects before discontinuation. Data on total fat mass measured by DXA were available from only sixty-three (65\%) children; the remaining children refused to undergo the second assessment. Compliance was similar in the experimental and control groups (approximately $90 \%$ of the empty sachets were returned after the intervention period).

The baseline demographic and clinical characteristics did not differ between the groups (Table 1). All outcome measures, including within-group and between-group differences, are summarised in Table 2. At 12 weeks, the BMI-for-age $z$-score difference did not differ between the experimental ( $n$ 40) and control ( $n$ 39) groups (MD 0.002, 95\% CI - 0.11, $0 \cdot 1)$. There were also no significant differences between the groups with regard to any of the secondary outcomes, such as percentage of body weight reduction (MD $-0 \cdot 57,95 \% \mathrm{CI}$ $-2 \cdot 34,1 \cdot 20$ ), mean BMI-for-age $z$-score (MD 0.14, 95\% CI $-0 \cdot 16,0 \cdot 44)$, self-reported energy intake $(P=0 \cdot 06)$, number of children with a BMI-for-age $<85$ th percentile (RR 0.33, $95 \%$ CI $0.01,8.43$ ), number of children with dyslipidaemias

Table 1. Baseline characteristics of the study groups

(Mean values and standard deviations; medians and ranges)

\begin{tabular}{|c|c|c|c|c|}
\hline & \multicolumn{2}{|c|}{$\begin{array}{l}\text { Oligofructose } \\
\qquad(n 48)\end{array}$} & \multicolumn{2}{|c|}{$\begin{array}{c}\text { Placebo } \\
(n 49)\end{array}$} \\
\hline & Mean & SD & Mean & SD \\
\hline Age (years) & $12 \cdot 3$ & 2.9 & $12 \cdot 4$ & $2 \cdot 7$ \\
\hline Body weight (kg) & $70 \cdot 1$ & $21 \cdot 3$ & $70 \cdot 7$ & $22 \cdot 0$ \\
\hline Height $(\mathrm{cm})$ & $155 \cdot 8$ & $15 \cdot 2$ & 157 & $14 \cdot 3$ \\
\hline BMI $\left(\mathrm{kg} / \mathrm{m}^{2}\right)$ & $28 \cdot 1$ & $4 \cdot 1$ & $27 \cdot 9$ & 4.4 \\
\hline Female $(n)$ & \multicolumn{2}{|c|}{20} & \multicolumn{2}{|c|}{28} \\
\hline Male $(n)$ & \multicolumn{2}{|c|}{28} & \multicolumn{2}{|c|}{21} \\
\hline Overweight $(n)$ & \multicolumn{2}{|c|}{8} & \multicolumn{2}{|c|}{8} \\
\hline Obese $(n)$ & \multicolumn{2}{|c|}{40} & \multicolumn{2}{|c|}{41} \\
\hline \multicolumn{5}{|c|}{$\begin{array}{l}\text { Total body fat measured } \\
\text { by DXA (g) }\end{array}$} \\
\hline Median & \multicolumn{2}{|c|}{28292} & \multicolumn{2}{|c|}{27157} \\
\hline Range & \multicolumn{2}{|c|}{$8802-56836$} & \multicolumn{2}{|c|}{$14529-54371$} \\
\hline
\end{tabular}

DXA, dual-energy X-ray absorptiometry. 
Table 2. Effect of oligofructose and placebo on outcome measures

(Mean values and standard deviations; number of patients and percentages; medians and ranges)

\begin{tabular}{|c|c|c|c|c|c|}
\hline & \multicolumn{2}{|c|}{ Oligofructose ( $n$ 40) } & \multicolumn{2}{|c|}{ Placebo ( $n$ 39) } & \multirow[b]{2}{*}{$P^{*}$} \\
\hline & $n$ & $\%$ & $n$ & $\%$ & \\
\hline \multicolumn{6}{|l|}{ Primary outcome } \\
\hline BMI-for-age $z$-score difference at 12 weeks & & & & & 0.97 \\
\hline Mean & \multicolumn{2}{|c|}{-0.247} & \multicolumn{2}{|c|}{-0.249} & \\
\hline SD & \multicolumn{2}{|c|}{0.24} & \multicolumn{2}{|c|}{0.2} & \\
\hline \multicolumn{6}{|l|}{ Secondary outcomes (at 12 weeks) } \\
\hline \multicolumn{6}{|l|}{ BMI-for-age $z$-score } \\
\hline \multicolumn{6}{|l|}{ Baseline } \\
\hline Mean & \multicolumn{2}{|c|}{$2 \cdot 7$} & \multicolumn{2}{|c|}{2.5} & \\
\hline SD & \multicolumn{2}{|c|}{0.7} & \multicolumn{2}{|c|}{0.6} & \\
\hline At 12 weeks & & & & & 0.37 \\
\hline Mean & \multicolumn{2}{|c|}{2.49} & & & \\
\hline $\mathrm{SD}$ & & & & & \\
\hline$P$ (baseline $v .12$ weeks) & & & & & \\
\hline Percentage of body weight reduction & & & & & 0.53 \\
\hline Mean & & & & & \\
\hline SD & & & & & \\
\hline Difference in total body fat (DXA; $g$ ) & & & & & 0.24 \\
\hline Mean & & & & & \\
\hline SD & & & & & \\
\hline Children with a BMI-for-age $<85$ th percentile & & & & & \\
\hline Baseline & 0 & 0 & 0 & & \\
\hline At 12 weeks & 0 & 0 & 1 & $2 \cdot 5$ & 0.49 \\
\hline$P$ (baseline $v .12$ weeks) & & & & & \\
\hline Energy intake $(\mathrm{kJ} / \mathrm{d})$ & & & & & \\
\hline Baseline & & & & & \\
\hline Median & & & & & \\
\hline Range & & & & & \\
\hline At 12 weeks & & & & & 0.06 \\
\hline Median & & & & & \\
\hline Range & & & & & \\
\hline$P$ (baseline $v .12$ weeks) & & & & & \\
\hline Children with glucose intolerance & & & & & \\
\hline Baseline & 0 & 0 & 1 & 2 & \\
\hline At 12 weekst & 0 & 0 & 0 & 0 & \\
\hline$P$ (baseline $v .12$ weeks) & & & & & \\
\hline Children with dyslipidaemias & & & & & \\
\hline Baseline & 12 & 25 & 13 & 26 & \\
\hline At 12 weeks $\dagger$ & 6 & 15 & 7 & 18 & 0.72 \\
\hline$P$ (baseline $v .12$ weeks) & & & & & \\
\hline Children with hypertension & & & & & \\
\hline Baseline & 4 & 8 & 1 & 2 & \\
\hline At 12 weeks $†$ & 2 & 5 & 0 & 0 & 0.49 \\
\hline$P$ (baseline $v .12$ weeks) & & & & & \\
\hline Physical activity (h/week) & & & & & \\
\hline Baseline & & & & & \\
\hline Median & & & & & \\
\hline Range & & & & & \\
\hline At 12 weeks & & & & & 0.36 \\
\hline Median & & & & & \\
\hline Range & & & & & \\
\hline$P$ (baseline $v .12$ weeks) & & & & & \\
\hline Secondary outcomes (at 24 weeks) & & & & & \\
\hline BMI $z$-score differences & & & & & 0.36 \\
\hline Mean & & & & & \\
\hline SD & & & & & \\
\hline BMI $z$-score & & & & & 0.04 \\
\hline Mean & & & & & \\
\hline SD & & & & & \\
\hline Children with a BMI-for-age $<85$ th percentile & 1 & 3 & 1 & 4.5 & 0.84 \\
\hline
\end{tabular}

DXA, dual-energy X-ray absorptiometry.

${ }^{*} P$ value for a comparison between the oligofructose and placebo groups.

† Measured only in children with abnormalities at baseline. 
Table 3. Adverse effects reported during the intervention (Number of patients and percentages)

\begin{tabular}{|c|c|c|c|c|c|}
\hline & \multicolumn{2}{|c|}{$\begin{array}{l}\text { Oligofructose } \\
\qquad(n 40)\end{array}$} & \multicolumn{2}{|c|}{$\begin{array}{c}\text { Placebo } \\
\text { (n 39) }\end{array}$} & \multirow[b]{2}{*}{$P$} \\
\hline & $n$ & $\%$ & $n$ & $\%$ & \\
\hline Abdominal pain & 12 & 30 & 5 & $12 \cdot 8$ & 0.08 \\
\hline Flatulence & 8 & 20 & 6 & $15 \cdot 4$ & 0.59 \\
\hline Diarrhoea/loose stool & 4 & 10 & 7 & $17 \cdot 9$ & 0.32 \\
\hline Borborygmi & 3 & $7 \cdot 5$ & 1 & $2 \cdot 6$ & 0.34 \\
\hline Feeling of fullness & 3 & 7.5 & 2 & $5 \cdot 1$ & 0.66 \\
\hline Loss of appetite & 3 & 7.5 & & D & 0.08 \\
\hline Nausea & 2 & 5 & & D & 0.16 \\
\hline Heartburn & 1 & 2.5 & & D & 0.32 \\
\hline
\end{tabular}

(RR 0.84, 95\% CI 0.31, 2.27), number of children with hypertension (RR 4.88, 95\% CI 0.24, 98.47), and physical activity $(P=0.36)$. Only one child presented with glucose intolerance at baseline; however, glucose tolerance improved after the intervention. In a subset of participants for whom data were available for analysis, there was also no significant difference between the groups with regard to the difference in total body fat measured by DXA $(P=0 \cdot 24)$. Adverse effects were similar in both groups (RR 1.65, 95\% CI 0.98, 2.79) (Table 3).

Within-group analyses (baseline $v .12$ weeks) showed no differences with one exception. There was a significant reduction in energy intake in the oligofructose $(P=0.02)$ and placebo $(P=0 \cdot 008)$ groups (Table 2$)$.

At 24 weeks, data from fifty-one (53\%) children were available for analysis. There was no significant difference between the groups with regard to anthropometric measurements after the follow-up period. The BMI-for-age $z$-score difference did not differ between the oligofructose and control groups (MD $-0.08,95 \%$ CI $-0.25,0.09$ ). The mean BMI-for-age $z$-score was also similar in both groups (MD $-0 \cdot 18$, 95\% CI -0.65 , $0 \cdot 25)$, as well as the number of children with a BMI-for-age $<85$ th percentile (RR $0.76,95 \%$ CI $0.05,11 \cdot 47$ ).

\section{Discussion}

The results of the present study showed that 12 weeks of supplementation with oligofructose, a soluble inulin-type fructan with known prebiotic properties, was not effective for body weight reduction in overweight and obese schoolaged children. The study groups did not differ with regard to anthropometric measurements (BMI-for-age $z$-score difference, mean BMI-for-age $z$-score and percentage of body weight reduction) and total body fat measured by DXA after the intervention. Energy intake assessed using a $3 \mathrm{~d}$ food record after the experimental period was similar in both groups. There were also no significant differences between the groups with regard to the prevalence of obesityrelated co-morbidities such as dyslipidaemia and hypertension. Adverse effects were comparable in both groups.

The major strength of the study is its design; a RCT is the design of choice for intervention studies. Appropriate methods were used to generate allocation sequence and allocation concealment. Follow-up was adequate at 12 weeks.
Data obtained from $81 \%$ of the participants were available for analysis. All these features minimise the risk of systemic bias.

However, the present study has some limitations. In contrast to the 12 -week follow-up visit, only $53 \%$ of the participants were available at the 24-week follow-up visit. However, as there was no effect of oligofructose supplementation at 12 weeks, it would be unrealistic to expect such an effect after stopping the intervention. The lack of perfect blinding is another potential limitation of the present study. The participants received oligofructose or placebo (both products were white powders) in identical sachets prepared by the hospital pharmacy. However, some participants reported that the study product became lumpy after being stored at room temperature. After unblinding, it was discovered that the problem was linked to oligofructose supplementation only. Another limitation is the assessment of compliance, which was not ideal. The study participants were asked to only return empty sachets; however, it would have been more appropriate to ask for half-empty as well as full sachets. Despite oligofructose clumping, compliance was similar in both groups. The biochemical laboratory examinations were performed only in children with abnormal values at the initial testing. This was based on current recommendations to test after 6 months of dietary intervention if abnormalities exist $^{(25)}$. However, one may argue that this is a limitation, as it does not allow one to identify children who initially tested healthy but whose health deteriorated over the course of the study. Only a very small number of children were included in these analyses. Given these considerations, caution is needed when interpreting data on hypertension, glucose intolerance and dyslipidaemias. A self-reported physical activity questionnaire was used to assess physical activity. Although it is convenient and cheap to use, there are some well-known limitations to its use, including item interpretation, recall and social desirability effects ${ }^{(26)}$.

To our knowledge, this is the first study to assess the effect of oligofructose supplementation on body weight in overweight and obese children. The results of the present study are in contrast to the findings of one RCT conducted in overweight and obese adults ${ }^{(14)}$. This trial, involving forty-eight subjects, showed that compared with placebo (maltodextrin), oligofructose supplementation $(21 \mathrm{~g} / \mathrm{d})$ resulted in a significant body weight reduction after a 12 -week intervention period. The researchers also assessed energy intake every 3 weeks during the experimental period. They reported no significant differences between the experimental and placebo groups with one exception. By week 6, a 29\% reduction in total energy intake was reported in the oligofructose group compared with the control group $(P=0.002)$. In the present study, the same study product was used for the same duration; however, it was used at a lower dose. The latter may be one of the explanations for the lack of an effect of oligofructose supplementation in the present study. In one double-blind, cross-over RCT conducted in thirty-one subjects (mixed population with normal-weight and overweight adults), no significant differences were observed in body weight after treatment with oligofructose ( 10 or $16 \mathrm{~g} / \mathrm{d}$ for $13 \mathrm{~d}$ ) compared with the placebo (maltodextrin). There was also no difference 
in appetite sensations and energy intake among the three treatment groups $^{(9)}$.

At least one recent study confirms that the dosage may be of importance. This is a 5-week escalating study performed by Pedersen et $a l^{(27)}$, who found oligofructose supplementation in the home environment at doses of $35 \mathrm{~g} / \mathrm{d}$ or higher to suppress hunger, although it did not affect energy intake. Moreover, it was found that oligofructose increased the concentrations of peptide YY and decreased those of pancreatic polypeptide, but did not influence the appetite profile during the appetite study session. The authors suggested a need for high doses of oligofructose to influence appetite.

Appetite was not assessed in the present study. However, Cani et al. ${ }^{(28)}$ found that consumption of oligofructose $(16 \mathrm{~g} / \mathrm{d})$ compared with placebo (dextrin maltose) significantly increased satiety following breakfast and dinner and also reduced hunger and prospective food consumption following dinner. No significant differences were observed in the remaining tests performed after breakfast and dinner and in all tests performed after lunch. Simultaneously measured energy intake was lower by $5 \%(P<0.05)$ after oligofructose supplementation compared with placebo. The authors hypothesised that oligofructose promotes satiety and reduces energy intake by influencing gut hormones involved in appetite regulation. However, no effect of oligofructose on appetite sensations and energy intake was found in another $\mathrm{RCT}^{(9)}$.

Some studies have evaluated the effect of the administration of oligofructose and inulin. In one RCT involving ninety-seven non-obese adolescents aged 9-13 years, a significant reduction was observed in body weight $(1.3$ (SD 0.6$) \mathrm{kg}$, $P=0.048)$, BMI difference $\left(0.52(\right.$ SD 0.16$\left.) \mathrm{kg} / \mathrm{m}^{2}, P=0.016\right)$ and BMI $z$-score difference $(0.13$ (SD 0.06$), P=0.048$ ) in the oligofructose plus inulin $(8 \mathrm{~g} / \mathrm{d})$ group compared with the control (maltodextrin) group after 1 year ${ }^{(29)}$. Notably, in this study, the anthropometric measurements were the secondary outcomes. The study was planned to assess the effect of inulin-type fructans on Ca absorption. Compared with the present study, the dose of oligofructose was similar; however, the duration of the intervention was significantly longer. Whether the longer administration and/or addition of inulin may explain the beneficial effect remains unclear.

In one recent RCT carried out in thirty obese women, treatment with an oligofructose/inulin 50/50 mix or placebo (maltodextrin) for 3 months was found to have no effects on BMI and the waist:hip ratio. However, these were the secondary outcomes; the study was not designed and powered sufficiently to demonstrate a difference if one actually exists. The primary endpoint of this study was to assess the prebiotic effect of inulin-type fructans on gut microbiota composition ${ }^{(30)}$. The effect of fructo-oligosaccharide supplementation on body weight was assessed in three RCT. Fructo-oligosaccharides are inulin-type fructans consisting of short chains of maximally five monomeric units, while oligofructose has chains with up to ten units. In the first cross-over RCT, involving twelve normal-weight adults, it was found that compared with consumption of cookies enriched with sucrose, consumption of cookies enriched with short-chain fructo-oligosaccharides $(20 \mathrm{~g} / \mathrm{d})$ for 4 weeks had no effect on body weight ${ }^{(31)}$.
In the second cross-over RCT involving thirty normal-weight and overweight adults also, no difference was observed after 2 months of short-chain fructo-oligosaccharide $(10.6 \mathrm{~g} / \mathrm{d})$ supplementation compared with placebo (maltodextrin plus aspartame) ${ }^{(32)}$. In contrast, in one RCT conducted in thirty-five obese women, a significant difference was found between the fructo-oligosaccharide group ( $0 \cdot 14 \mathrm{~g} / \mathrm{kg}$ per $\mathrm{d}$ for 4 months) and the placebo group with respect to body weight (MD $-16.1 \mathrm{~kg}, P<0.05$ ) and BMI (MD $-4.0 \mathrm{~kg} / \mathrm{m}^{2}$, $P<0.05)^{(33)}$. Finally, two RCT investigated the effect of inulin. Both reported no significant difference in body weight between the inulin groups $(10 \mathrm{~g} / \mathrm{d})$ treated for 2 months ${ }^{(34)}$ or 3 months ${ }^{(35)}$ and their respective control groups.

Data from the literature suggest that adverse effects, especially side gastrointestinal effects, occur more often in subjects receiving fructans with a shorter chain such as oligofructose $^{(36)}$. In the present study, adverse effects were similar in both groups. However, no standardised measurement instrument was used for adverse events, and the study lacks power to determine adverse effects.

\section{Conclusion}

Our findings do not support the hypothesis that oligofructose supplementation, as dosed in the present study, is effective for body weight reduction in overweight and obese school-aged children.

\section{Acknowledgements}

The present study was financially supported by a research grant (grant no. RG 4/2011) from the Fundacja Nutricia (Nutricia Foundation). Fundacja Nutricia had no role in the design and analysis of the study or in the writing of this article.

The authors' contributions are as follows: H. S. initially conceptualised the study. Both authors contributed to the study protocol. A. L. was responsible for data collection, data analysis, data interpretation, and manuscript preparation. A. L. had primary responsibility for writing the first draft of the manuscript. Both authors contributed to (and agreed upon) the final version.

The authors declare no conflicts of interest.

\section{References}

1. World Obesity Federation (2012) About obesity. http://www. worldobesity.org/aboutobesity/ (accessed September 2014).

2. Bäckhed F, Ding H, Wang T, et al. (2004) The gut microbiota as an environmental factor that regulates fat storage. Proc Natl Acad Sci U S A 101, 15718-15723.

3. Ley RE, Turnbaugh PJ, Klein S, et al. (2006) Microbial ecology: human gut microbes associated with obesity. Nature 444, 1022-1023.

4. Hildebrandt MA, Hoffmann C, Sherrill-Mix SA, et al. (2009) High-fat diet determines the composition of the murine gut microbiome independently of obesity. Gastroenterology 137, 1716-1724.

5. Cani PD, Amar J, Iglesias MA, et al. (2007) Metabolic endotoxemia initiates obesity and insulin resistance. Diabetes 56, 1761-1772. 
6. Niness KR (1999) Inulin and oligofructose: what are they? J Nutr 129, 1402-1406.

7. Cani PD, Neyrinck AM, Maton N, et al. (2005) Oligofructose promotes satiety in rats fed a high-fat diet: involvement of glucagon-like peptide-1. Obes Res 13, 1000-1007.

8. Cani PD, Dewever C \& Delzenne NM (2004) Inulin-type fructans modulate gastrointestinal peptides involved in appetite regulation (glucagon-like peptide-1 and ghrelin) in rats. Br J Nutr 92, 521-526.

9. Verhoef SP, Meyer D \& Westerterp KR (2011) Effects of oligofructose on appetite profile, glucagon-like peptide 1 and peptide YY3-36 concentrations and energy intake. Br J Nutr 106, 1757-1762.

10. Cani PD, Lecourt E, Dewulf EM, et al. (2009) Gut microbiota fermentation of prebiotics increases satietogenic and incretin gut peptide production with consequences for appetite sensation and glucose response after a meal. Am J Clin Nutr 90, 1236-1243.

11. Liber A \& Szajewska H (2013) Effects of inulin-type fructans on appetite, energy intake, and body weight in children and adults: systematic review of randomized controlled trials. Ann Nutr Metab 63, 42-54.

12. Bonsu NK, Johnson CS \& McLeod KM (2011) Can dietary fructans lower serum glucose? J Diabetes 3, 58-66.

13. Brighenti F (2007) Dietary fructans and serum triacylglycerols: a meta-analysis of randomized controlled trials. J Nutr 137, 2552-2556.

14. Parnell JA \& Reimer RA (2009) Weight loss during oligofructose supplementation is associated with decreased ghrelin and increased peptide $\mathrm{YY}$ in overweight and obese adults. Am J Clin Nutr 89, 1751-1759.

15. World Health Organization (2007) Growth Reference Data for 5-19 years. Geneva: World Health Organization. http://www.who.int/growthref/who2007_bmi_for_age/en/ index.html (accessed May 2014).

16. Griffin IJ, Davila PM \& Abrams SA (2002) Non-digestible oligosaccharides and calcium absorption in girls with adequate calcium intakes. BrJ Nutr 87, 187-191.

17. Van den Heuvel EG, Muys T, van Dokkum W, et al. (1999) Oligofructose stimulates calcium absorption in adolescents. Am J Clin Nutr 69, 544-548.

18. Jarosz M (editor) (2008) Normy żywienia cztowieka. Podstawy prewencji otytości $i$ chorób niezakaźnych (Standards of Human Nutrition. Principles of Preventing Obesity and Non-communicable Diseases). Warszawa: PZWL.

19. Craig CL, Marschall AL, Sjostrom M, et al. (2003) International physical activity questionnaire: 12 -country reliability and validity. Med Sci Sports Exerc 35, 1381-1395.

20. Pludowski P, Litwin M, Sladowska J, et al. (2008) Bone mass and body composition in children and adolescents with primary hypertension: preliminary data. Hypertension $\mathbf{5 1}$, 77-83.

21. World Health Organization (2006) Definition and Diagnosis of Diabetes Mellitus and Intermediate Hyperglycemia: Report of a World Health Organization/International Diabetes Federation Consultation. Geneva: World Health Organization. http://www.who.int/diabetes/publications/ Definition\%20and\%20diagnosis\%20of\%20diabetes_new.pdf (accessed May 2014).
22. Daniels SR, Greer FR \& Committee on Nutrition (2008) Lipid screening and cardiovascular health in childhood. Pediatrics 122, 198-208.

23. Lurbe E, Cifkova R, Cruickshank JK, et al. (2009) European Society of Hypertension. Management of high blood pressure in children and adolescents: recommendations of the European Society of Hypertension. J Hypertens 27 , 1719-1742.

24. Ford AL, Bergh C, Södersten P, et al. (2009) Treatment of childhood obesity by retraining eating behaviour: randomised controlled trial. BMJ 340, b5388.

25. Expert Panel on Integrated Guidelines for Cardiovascular Health and Risk Reduction in Children and Adolescents; National Heart, Lung, and Blood Institute (2011) Expert panel on integrated guidelines for cardiovascular health and risk reduction in children and adolescents: summary report. Pediatrics 128, 213-256.

26. Loprinzi PD \& Cardinal B (2011) Measuring children's physical activity and sedentary behaviors. J Exerc Sci Fit 13, 15-23.

27. Pedersen C, Lefevre S, Peters V, et al. (2013) Gut hormone release and appetite regulation in healthy non-obese participants following oligofructose intake. A dose-escalation study. Appetite 66, 44-53.

28. Cani PD, Joly E, Horsmans Y, et al. (2006) Oligofructose promotes satiety in healthy human: a pilot study. Eur $J$ Clin Nutr 60, 567-572.

29. Abrams SA, Griffin IJ, Hawthorne KM, et al. (2007) Effect of prebiotic supplementation and calcium intake on body mass index. J Pediatr 151, 293-298.

30. Dewulf EM, Cani PD, Claus SP, et al. (2013) Insight into the prebiotic concept: lessons from an exploratory, double blind intervention study with inulin-type fructans in obese women. Gut 62, 1112-1121.

31. Luo J, Rizkalla SW, Alamowitch C, et al. (1996) Chronic consumption of short-chain fructooligosaccharides by healthy subjects decreased basal hepatic glucose production but had no effect on insulin-stimulated glucose metabolism. Am J Clin Nutr 63, 939-945.

32. Giacco R, Clemente G, Luongo D, et al. (2004) Effects of short-chain fructo-oligosaccharides on glucose and lipid metabolism in mild hypercholesterolaemic individuals. Clin Nutr 23, 331-340.

33. Genta S, Cabrera W, Habib N, et al. (2009) Yacon syrup: beneficial effects on obesity and insulin resistance in humans. Clin Nutr 28, 182-187.

34. Jackson KG, Taylor GR, Clohessy AM, et al. (1999) The effect of the daily intake of inulin on fasting lipid, insulin and glucose concentrations in middle-aged men and women. Br J Nutr 82, 23-30.

35. Tovar AR, Caamaño MD, García-Padilla S, et al. (2012) The inclusion of a partial meal replacement with or without inulin to a calorie restricted diet contributes to reach recommended intakes of micronutrients and decrease plasma triglycerides: a randomized clinical trial in obese Mexican women. Nutr J 11, 44.

36. Kelly G (2008) Inulin-type prebiotics - a review: part 1. Altern Med Rev 13, 315-329. 